\title{
Effect of conduction block in classification and prognosis of Guillain-Barre syndrome
}

\author{
Yu-Chen Wang, Guo-Dong Feng, Jing Wang, Xue-Dong Liu, Gang Zhao \\ Department of Neurology, Xijing Hospital, The Fourth Military Medical University, Xi'an 710032, Shaanxi, China.
}

\section{A B S T R A C T}

Aim: The aim was to investigate the electro-physiological characteristics in disease progression of Guillain-Barre syndrome (GBS) and observe the effect of conduction block (CB) in classification and severity of the disease. Methods: Two hundred and ninety-four patients with GBS were divided into acute inflammatory demyelinating poly-neuropathy (AIDP) group, acute motor axonal neuropathy (AMAN) group and equivocal group according to their electro-physiological results and then reclassificated after electro-physiological review. All of the patients were followed for 6 months since their attacks. Results: Bad prognosis is more pronounced in AMAN group than in AIDP group $(P<0.05)$. Most of the patients classificated as AIDP transformed into AMAN when CB occurred in the early phase of the disease. There is a positive relationship between $\mathrm{CB}$ in the early phase of the disease and severity of illness $(P<0.05)$, but $\mathrm{CB}$ showed no correlation with prognosis of the patients $(P>0.05)$. Conclusion: $\mathrm{CB}$ in the early phase of GBS indicates the probability of AIDP transforming into AMAN; it suggests that patients with $\mathrm{CB}$ in the early phase of the disease might be in serious conditions in a certain extent.

Key words: Clinical features, Guillain-Barre syndrome, nerve conduction block, nerve electrophysiology

\section{INTRODUCTION}

Gillan-Barre syndrome (GBS), which is also known as acute inflammatory demyelinating polyradiculoneuropathy, is an autoimmune disease in which the typical clinical symptoms are rapidly progressing symmetrical weakness, areflexia and cerebrospinal fluid (CSF) protein levels elevated without accompanying pleocytosis. It usually affects spinal nerve roots especially the anterior roots, ganglions and peripheral nerves, sometimes affects the cranial nerves.

According to its clinical manifestation, laboratory examinations and electro-physiological characteristics, GBS can be classified as acute inflammatory demyelinating poly-neuropathy (AIDP), acute motor axonal neuropathy (AMAN), acute motor sensory axonal neuropathy, Miller-Fisher syndrome (MFS), acute autonomic neuropathy, and acute sensory neuropathy. ${ }^{[1]}$ GBS is single-phase process and self-limiting, so most

\begin{tabular}{|l|l|}
\hline \multicolumn{2}{|c|}{ Access this article online } \\
\hline Quick Response Code: & \\
\hline & Website: \\
\hline & www.nnjournal.net \\
\cline { 2 - 2 } & DOI: \\
\hline
\end{tabular}

of the GBS patients have a good prognosis, but some may have a bad prognosis clinically. As the disease progresses, some of the AIDP patients may turn into AMAN; some patients without obvious curative effect may progress into chronic inflammatory demyelinating polyneuropathy; and some patients may transform into relapsing GBS. Neural electro-physiological examination, an irreplaceable auxiliary examination for diagnosis of GBS, can provide an important basis for the diagnosis and classification of the disease. In this study, the clinical data of 338 GBS patients who were hospitalized during the period of August 2008 to February 2013 were analyzed retrospectively.

\section{METHODS}

\section{Patients}

This study retrospectively includes 338 GBS patients who were hospitalized during the period of August 2008 to February 2013. All the cases were diagnosed under the diagnosis and treatment guidelines of GBS published in 2010 by Chinese medicine association. Excluding 20 cases without neural electro-physiological examination and 24 patients who were diagnosed with MFS, 294 cases were included in this study eventually, with 186 male patients (63.3\%) and 108 female patients (36.7\%). Patient's ages ranged from 4 to 82 years (mean $40.4 \pm 18.3$ years), and the average hospital stay was 18.8 days. There

Corresponding Author: Dr. Yu-Chen Wang, Department of Neurology, Xijing Hospital, The Fourth Military Medical University, No.169 Changle West Road, Xi'an 710032, Shaanxi, China. E-mail: xianhhwangyc@hotmail.com 
were precursor events emerging 1-4 weeks before in some of the patients: respiratory infection or fever in 126 cases (42.9\%), digestive tract infection in 71 cases $(24.1 \%)$, flu vaccination in 3 patients $(1.0 \%)$, influenza vaccination in 2 cases $(0.7 \%)$, rabies vaccination in 2 cases $(0.7 \%)$, pregnancy in 1 case $(0.3 \%)$, and the rest cases with no precursor events. The first symptom was limb weakness in 276 cases (93.9\%), sensory disturbance in 98 cases (33.3\%), and cranial nerve symptom in 54 cases (18.4\%). There were 76 cases $(25.9 \%)$ with clinical symptoms of cranial nerve lesions, including 36 cases with facial nerve paralysis, 8 cases with ophthalmoplegia, 7 cases with diplopia and 62 cases with drinking water choking. There were 102 cases (34.7\%) with clinical symptoms of disturbance of sensation, 260 cases (88.4\%) with upper-limb weakness, 276 cases (93.9\%) with lower limb weakness. There were 192 cases (65.3\%) with tendon hyporeflexia, 66 cases (22.4\%) with disappearing and 11 cases $(3.7 \%)$ with hyperreflexia. 261 cases had a lumbar puncture while they were hospitalized, and there were 205 cases (78.5\%) with albumincytological dissociation. Two hundred and eighteen cases had CSF immunoglobulin test, and there were 178 cases (81.7\%) with high levels of $\operatorname{IgG}, 36$ cases (16.5\%) with high levels of IgM, 137 cases (62.8\%) with high levels of IgA. The average time to disease peak period was 10 days; 65 cases (22.1\%) suffered from the lung infection while 16 cases (5.4\%) suffered from urinary tract infection.

\section{Electro-physiological examination}

Keypoint electromyography made by the Danish Dandi Company was used. Since early stage of the disease, the electro-physiological result showed abnormal F-wave only, and the result of nerve conduction showed reduction of compound muscle action potential in most cases, so all patients were performed at least one electro-physiological examination 2 weeks after the onset of disease. Of the patients, 132 cases had their first electro-physiological examination 2 weeks after the onset of disease and had another check within 10-14 days after the first check: (1) All patients had nerve conduction test by using surface electrodes to record and stimulate. We detected motor conductive test on bilateral median nerve, ulnar nerve, peroneal nerve, and tibial nerve recording the motor nerve conduction velocity, distal latency, and amplitude, etc., and observed whether there was a nerve conduction block (CB) or not. Sensory conduction test were performed at median nerve, ulnar nerve, sural nerve, and phil shallow nerve recording the sensory nerve conduction velocity; (2) all patients had F-wave detection on the median nerve, ulnar nerve peroneal nerve and tibial nerve by recording the wave rate, latency, etc.; (3) needle electromyography were performed on thenar muscle, hypothenar muscle, deltoid, quadriceps, pretibial muscle, and gastrocnemius by observing whether there was a spontaneous activity or not on the resting moment and by testing the motor unit potential and recruitment order of slight and strong muscle contraction. ${ }^{[2,3]}$

\section{Diagnostic and evaluation criteria}

(1) GBS and nerve block were diagnosed under the diagnosis and treatment guidelines of GBS published in 2010 by Chinese Medicine Association; (2) the neural electro-physiological types were classified into AIDP and AMAN according to the electro-physiological diagnostic standard [Table 1];[4] (3) normal patients and the patients not satisfying the diagnostic criteria of AIDP and AMAN were included into the unclear type group; (4) the disease classification and followup results were marked according to rating scales designed by Hughes et al. Based on patients' ability to walk with the help, patients were classified into mild type and serious type. Patients with Hughes score equal or lesser than two points is the mild type, and equal or more than three points is a serious type; (5) the prognostic evaluations were marked according to rating scales designed by Hughes et al. Based on the patients' sequelaes (whether can walk without help or not), patients were classified into favorable prognosis type and poor prognosis type. Patients with Hughes score equal or lesser than two points is the favorable prognosis type and equal or more than 3 points is the poor prognosis type; (6) follow-up: 294 cases of GBS patients included in this study were follow-up by telephone for 6 months after being discharged from hospital and 103 cases were lost to follow-up, so the response rate was $65.0 \%$. Among the remaining 191 cases, 3 died of non-GBS cause. There were 188 cases with efficient results, which were included in this study eventually.

\section{Statistical analysis}

Data analysis was carried out using SPSS 17.0 Statistical Analysis Software (Polar Enginneering and Consulting. http://www.winwrap.com/). We compared clinical symptom, grading and prognosis between groups with Chi-squared test, and considered statistical significance at $P<0.05$.

\begin{tabular}{|c|c|c|}
\hline Types & $\begin{array}{l}\text { Electro-physiological } \\
\text { index }\end{array}$ & Diagnostic standard \% \\
\hline AIDP & $\begin{array}{l}\text { Conduction velocity } \\
\text { far-end lurk waveform } \\
\text { disperse F wave }\end{array}$ & $\begin{array}{l}\text { At least } 2 \text { nerves have } 1 \text { or more } \\
\text { electro-physiological behaviors } \\
\text { < } 90 \text { LLN; if CMAP < } 50 \text { LLN; } \\
\text { < } 85 \text { LLN; > } 110 \text { ULN; if CMAP < LLN; } \\
\text { > } 120 \text { ULN; clear waveform disperse } \\
\text { > } 120 \text { ULN }\end{array}$ \\
\hline AMAN & & $\begin{array}{l}\text { No electro-physiological behaviors of } \\
\text { AIDP; } 2 \text { or more nerves CMAP }<20\end{array}$ \\
\hline
\end{tabular}




\section{RESULTS}

Neural electro-physiological results

There were 102 cases (34.7\%) in AIDP group and 81 cases (27.6\%) in AMAN group [Figure 1]. Based on the first electro-physiological testing, 132 patients were classified into: 58 cases (43.9\%) of AIDP, 24 cases (18.2\%) of AMAN, 50 cases (37.9\%) of unclear [Figure 2]. Cases belonged to AMAN group based on two different testing results were fewer than the cases in AIDP group and unclear classification cases group.

Relationship between early nerve conduction block and its electro-physiological changes

The first electro-physiological results for 132 cases with rechecks were: 58 cases (44\%) in AIDP group, 24 cases (18\%) in AMAN group, 50 cases (38\%) in unclear classification group [Figure 3]. A total of 36 cases in AIDP group had CB, and cases transforming into AIDP and AMAN were 19 and 17, respectively.

Relationship between different types and the prognosis The first electro-physiological results and the recheck results all demonstrated that comparing to AIDP, AMAN had more cases with poor prognosis [Tables 2 and 3] (all $P<0.05$ ).

Relationship between early nerve conduction block and the severity of the illness

Results demonstrated that the severity of the illness was related to the development of CB in early stage in AIDP group and unclear classification group (all $P<0.05$ ) [Table 4].

The results of Chi-squared test within each type of group were: in AIDP the value was 11.334, $P=0.001$, in unclear classification the value is $8.408, P=0.004$, both with statistical significance; in AMAN group the value is $3.472, P=0.062$, with no statistical difference.

Relationship between early nerve conduction block and prognosis

Results demonstrated that irrespective of the severity of the disease, poor prognosis was not related to the development of CB (all $P>0.05$ ) [Tables 5 and 6].

\section{DISCUSSION}

In this study, more male than female patients were included. Respiratory tract and intestinal infections were the most common precursor events. A few patients had influenza vaccine, H1N1 influenza vaccine and rabies vaccine before the onset of the illness. It has been reported that $\mathrm{H} 1 \mathrm{~N} 1$ vaccine maybe is a risk factor of GBS, but season influenza vaccine was not related to it. ${ }^{[5,6]}$ In our data, there is no evidence that $\mathrm{H} 1 \mathrm{~N} 1$ influenza vaccine was related to GBS. The most

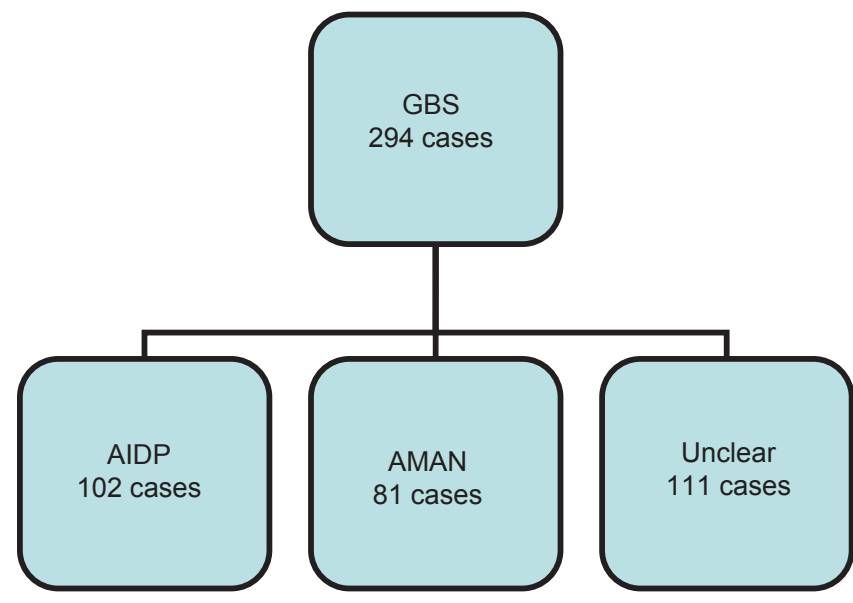

Figure 1: Neural electro-physiological results. GBS: Gillan-Barre syndrome; AIDP: acute inflammatory demyelinating poly-neuropathy; AMAN: acute motor axonal neuropathy

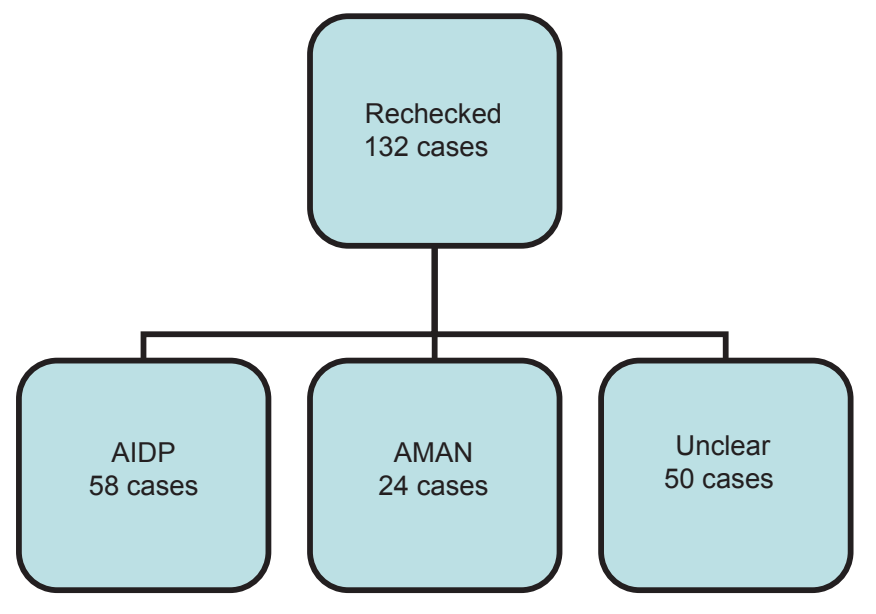

Figure 2: One hundred and thirty-two rechecked cases' first classification. AIDP: acute inflammatory demyelinating poly-neuropathy; AMAN: acute motor axonal neuropathy

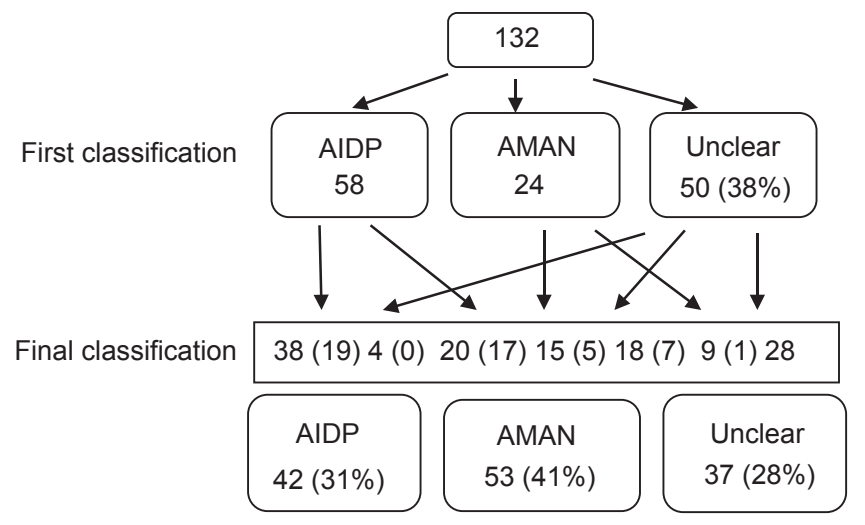

Figure 3: One hundred and thirty-two Gillan-Barre syndrome patients' first and final neural electro-physiological classifications (conduction block numbers in brackets clear to arrows). AIDP: acute inflammatory demyelinating poly-neuropathy; AMAN: acute motor axonal neuropathy

common symptoms were symmetrical limb weakness and numbness. Sensory disturbance is usually milder than motor disturbance with reducing or disappearing tendon reflex. The common cranial nerve damages are facial nerve paralysis, drinking water choking, hoarseness, and ophthalmoplegial. All the cases were followed by telephone for 6 months after hospital 
discharge. The response rate was $65.0 \%$. Twenty-one percent of the patients had serous sequel which was the same as those reported in the literature. The mortality was $10.6 \%$ which was higher than previously reported. ${ }^{[6]}$

The most common subtypes of GBS are AIDP and AMAN with only motor fiber damage. According to the literature, in North America and Europe more than $90 \%$ of the patients is classified as AIDP, ${ }^{[4]}$ but in China, the most common subtype is AMAN, and $65 \%$ of the GBS are AMAN. ${ }^{[7]}$ In this study, most cases among the 294 GBS patients were AIDP. Of these, 132 cases were classified as AIDP based on their first electro-physiological examination. These findings are in contrast with what previously reported.

Because the classification based on early stage GBS electro-physiological results is inaccurate, electrophysiology testing was repeated after the illness developed and the percentage of AIDP and AMAN cases changed comparing to the early stage results. ${ }^{[8]}$ We classified patients into AIDP and AMAN groups and unclear classification group which included patients whose electro-physiological testing were normal and those who did not meet the diagnostic criteria of AIDP and AMAN. We found that during disease progression electro-physiological results, as well as the electro-physiological classification, changed. The percentage of AMAN patients after recheck increased significantly compared to the first check (from 18\% to 41\%), which is the same as reported literature, ${ }^{[8,9]}$ and AIDP reduced from $44 \%$ to $31 \%$. We also found that AMAN had a worse prognosis than AIDP, and this finding is consistent with the

\begin{tabular}{lccc|}
\hline Table 2: All cases classifications and prognosis & \\
\hline Prognosis & AIDP & AMAN & Total \\
\hline Good prognosis & 59 & 27 & 86 \\
Poor prognosis & 12 & 19 & 31 \\
Total & 71 & 46 & 117 \\
\hline
\end{tabular}

$\chi^{2}=8.535, P=0.003$. AIDP: acute inflammatory demyelinating poly-neuropathy; AMAN: acute motor axonal neuropathy

Table 3: Rechecked cases classifications and prognosis

\begin{tabular}{lccc}
\hline Prognosis & AIDP & AMAN & Total \\
\hline Good prognosis & 35 & 8 & 43 \\
Poor prognosis & 6 & 9 & 15 \\
Total & 41 & 17 & 58 \\
\hline
\end{tabular}

$\chi^{2}=9.197, P=0.002$. AIDP: acute inflammatory demyelinating poly-neuropathy; AMAN: acute motor axonal neuropathy literature, ${ }^{[10,11]}$ thus suggesting a poor prognosis in more patients. The classification based on early stage GBS electrophysiologic results only may lead to inaccurate judgment of the patients' diagnosis and prognosis, instead continuous electrophysiology recheck can reflect the change of patient's condition without delay.

Among the patients transforming from AIDP into AMAN, CB occurred in 17 cases in the early onset of the illness: 5 and 7 cases in AMAN and unclear classification group, respectively. CB is a blockage in a nerve that prevents impulses from being conducted across a given segment although the nerve beyond is viable and is one of the important electro-physiological parameters of peripheral nerve functional status. $\mathrm{CB}$ is one of the physiological results caused by demyelination and is also the basic physiological mechanism of most clinical manifestations. ${ }^{[12]}$ Most studies of CB published before are on multifocal motor neuropathy and amyotrophic lateral sclerosis, peroneal muscular atrophy and peripheral neuropathy caused by pressure. ${ }^{[13]}$ Though conventional wisdom holds that the main cause of CB is demyelination and CB is the typical characteristics of demyelinating, recent studies demonstrated that demyelination is not the only reason for CB. It can caused by demyelination, depolarization on node of ranvier nearby axolemma, hyperpolarization and sodium channel damage. ${ }^{[14]}$ The damage of nearby axolemma may cause CB, electro-physiological manifest as decreased amplitude, discretized waveform. If the illness continues to progress, reversible CB will turn into irreversible CB, and axonal degeneration. This might explain why some of the CB cases transformed into AMAN in electro-physiological classification.

Our study demonstrated that CB not only occurred in AIDP patients, but also in AMAN and unclear classification patients. In recent years, other groups found that CB plays an important role in axon damaged AMAN. ${ }^{[15,16]}$ Kuwabara et al. ${ }^{[17]}$ thought that the possible cause of CB in AMAN was axonal degeneration. The bridge type union of GM-1 antibody-mediated inflammatory cells and axons, the release of inflammatory mediators, local acidosis, damage on sodium ion channel of Axonal membrane and tight junction of axon myelin (some authors believe that this is a different type of demyelination from AIDP) resulted in a further decline of the safety factors, eventually leading to $\mathrm{CB} .{ }^{[18]}$

Table 4: Relationship between different classifications nerve CB and the severity in 294 cases

\begin{tabular}{|c|c|c|c|c|c|c|c|c|c|}
\hline \multirow[t]{2}{*}{ Severity } & \multicolumn{3}{|c|}{ AIDP } & \multicolumn{3}{|c|}{ AMAN } & \multicolumn{3}{|c|}{ Unclear } \\
\hline & With CB & Without CB & Total & With CB & Without CB & Total & With CB & Without CB & Total \\
\hline Slight & 6 & 18 & 24 & 2 & 17 & 19 & 2 & 24 & 26 \\
\hline Severe & 50 & 28 & 78 & 20 & 42 & 62 & 32 & 53 & 85 \\
\hline Total & 56 & 46 & 102 & 22 & 59 & 81 & 34 & 77 & 111 \\
\hline
\end{tabular}

AIDP: acute inflammatory demyelinating poly-neuropathy; AMAN: acute motor axonal neuropathy; CB: conduction block 
Table 5: Early CB and prognosis of 140 severe GBS cases

\begin{tabular}{lccc} 
Prognosis & With CB & Without CB & Total \\
\hline Good prognosis & 46 & 54 & 100 \\
Poor prognosis & 15 & 25 & 40 \\
Total & 61 & 79 & 140 \\
\hline
\end{tabular}

$\chi^{2}=0.840, P=0.360$. CB: conduction block; GBS: Gillan-Barre syndrome

\begin{tabular}{lccc}
\hline Table 6: CB and prognosis of 47 & slight $\mathrm{GBS}$ cases & \\
\hline Prognosis & With CB & Without CB & Total \\
\hline Good prognosis & 7 & 39 & 46 \\
Poor prognosis & 0 & 1 & 1 \\
Total & 7 & 40 & 47 \\
\hline
\end{tabular}

$\chi^{2}=0.179, P=0.672$. CB: conduction block, GBS: Gillan-Barre syndrome

Our results suggested that the severity of the illness was related to the development of CB in early stage in AIDP group and unclear classification group but in the AMAN group is limited by small sample size. As mentioned previously, damage factors of the axonal membrane may the cause of $\mathrm{CB}$, and then reversible $\mathrm{CB}$ will turn into irreversible $\mathrm{CB}$ and axonal degeneration. According to the report of Kokubun et al..$^{[9]}$, the proportion of the two outcomes is 1:1 on AMAN patients who had CB. Patients in this group with CB in early stage were not related to the severity of the illness may be because some patients had reversible CB. In addition, the use of immunoglobulin in early stage of disease in patients with serious conditions may improve the prognosis and the finding that development into CB in early stage correlates with the severity of the illness might be another factor.

Due to the objective condition limit that our patients mostly come from surrounding cities and counties, and even other provinces, it is difficult to diagnose these patients' neurological recovery face-to-face after they are discharged from our hospital. We have to perform telephone follow-up for most of them. At the same time, many patients filled temporary numbers in the contact information form when hospitalized, which were no longer used after they went back. This also limited the follow-up results, and the response rate was only $65 \%$. Furthermore, through telephone follow-up, we can't evaluate the neural function of patients completely and clearly. We will try to improve this in future work and research.

In conclusion, reversible $\mathrm{CB}$ might be the cause of changes in patients' electro-physiological classification. CB is not only a typical electro-physiological manifestation in AIDP, but also a manifestation of axonal degeneration for AMAN in the early stage. $\mathrm{CB}$ and axonal degeneration are caused by immunemediated damage factors which attack axon membrane on the motor fiber. To a certain extent, CB is very helpful in classifying the severity of the illness. ${ }^{[14]}$ Electrophysiology recheck can be very meaningful to reveal change of patient's condition, classification alteration and severity of the nerve damage in time.

\section{REFERENCES}

1. Dang JX. Electromyography Diagnosis and Clinical Applications. Beijing: People's Medical Publishing House; 2005.

2. Cui LY. Electromyography Condensed Handbook. Beijing: science Press; 2008.

3. Zhang KL, Xu JG. Clinical Utility of Electromyography Diagnosis Technology. Shanghai: fudan University Press; 2005.

4. Hadden RD, Cornblath DR, Hughes RA, Zielasek J, Hartung HP, Toyka KV, Swan AV. Electrophysiological classification of Guillain-Barré syndrome: clinical associations and outcome. Plasma Exchange/Sandoglobulin Guillain-Barré Syndrome Trial Group. Ann Neurol 1998;44:780-8

5. Lehmann HC, Hartung HP, Kieseier BC, Hughes RA. Guillain-Barré syndrome after exposure to influenza virus. Lancet Infect Dis 2010;10:643-51.

6. Yuki N, Hartung HP. Guillain-Barré syndrome. N Engl J Med 2012;366:2294-304.

7. Ho TW, Mishu B, Li CY, Gao CY, Cornblath DR, Griffin JW, Asbury AK, Blaser MJ, McKhann GM. Guillain-Barré syndrome in northern China. Relationship to Campylobacter jejuni infection and anti-glycolipid antibodies. Brain 1995;118:597-605.

8. Uncini A, Manzoli C, Notturno F, Capasso M. Pitfalls in electrodiagnosis of Guillain-Barré syndrome subtypes. J Neurol Neurosurg Psychiatry 2010;81:1157-63.

9. Kokubun N, Nishibayashi M, Uncini A, Odaka M, Hirata K, Yuki N. Conduction block in acute motor axonal neuropathy. Brain 2010;133:2897-908.

10. Hiraga A, Mori M, Ogawara K, Hattori T, Kuwabara S. Differences in patterns of progression in demyelinating and axonal Guillain-Barré syndromes. Neurology 2003;61:471-4.

11. Brown WF, Feasby TE, Hahn AF. Electrophysiological changes in the acute "axonal" form of Guillain-Barre syndrome. Muscle Nerve 1993;16:200-5.

12. Liu T, Lu ZN. Demyelinating neuropathy physiological basis of conduction block. Str Nerv Dis 2006;13:59.

13. Ghosh A, Donaghy M. Multifocal motor neuropathy. Neurol India 2002;50:408-16.

14. Tang XF. Current insights into nerve block. Chin JNeuo 2003;36:404

15. Kokubun N, Shahrizaila N, Hirata K, Yuki N. Conduction block and axonal degeneration co-occurring in a patient with axonal Guillain-Barré syndrome. J Neurol Sci 2012;319:164-7.

16. Raguer N, Gratacós M, Lladó E, Lara N, Seoane JL, Benito M, Rio J, Gámez J. Acute motor axonal neuropathy (AMAN), description of neurophysiological findings in 8 patients with early nerve conduction blocks. Clin Neurophysiol 2006;117:1-2.

17. Kuwabara S, Bostock H, Ogawara K, Sung JY, Kanai K, Mori M, Hattori T, Burke D. The refractory period of transmission is impaired in axonal Guillain-Barré syndrome. Muscle Nerve 2003;28:683-9.

18. Wang RB, Liu XZ. Nerve conduction block and its mechanism $J$ Brain Nerv Dis 2006;14:239.

Cite this article as: Wang YC, Feng GD, Wang J, Liu XD, Zhao G. Effect of conduction block in classification and prognosis of Guillain-Barre syndrome. Neuroimmunol Neuroinflammation 2014;1(2):77-81.

Source of Support: Nil. Conflict of Interest: No.

Received: 12-05-2014; Accepted: 18-07-2014 